\title{
Reduced up-regulation of memory and adhesion/integrin molecules in susceptible mice and poor expression of immunity to pulmonary tuberculosis
}

\author{
Veronica Gruppo, Oliver C. Turner, Ian M. Orme and Joanne Turner
}

Author for correspondence: Ian M. Orme. Tel: +1 970491 5777. Fax: +1 9704915125. e-mail: Ian.Orme@colostate.edu

Mycobacteria Research Laboratories, Department of Microbiology, Immunology and Pathology, Colorado State University, Fort Collins, CO 80523, USA

\begin{abstract}
Previous studies examining the expression of adhesion and integrin molecules on CD4 T lymphocytes generated in response to virulent Mycobacterium tuberculosis infection revealed that certain inbred mouse strains susceptible to breakdown of chronic disease and subsequent reactivation had poor expression of these molecules, which might underlie their inability to adequately focus into lung tissues and mediate protection. The current study examines the possibility that prior vaccination with BCG, or a prior tuberculosis infection, would overcome this deficiency. It was found, however, that this was not the case. Whereas both resistant (C57BL/6) and susceptible (DBA/2, CBA/J) strains were equally well protected in the spleen after intravenous challenge, the latter strains were poorly protected in the lungs regardless of whether the challenge was given by the intravenous or aerosol route. Again, this was associated with poor up-regulation of adhesion and integrin molecules and with histological evidence in memory immune animals of a reduced and delayed influx of $\mathrm{T}$ lymphocytes into the lungs.
\end{abstract}

Keywords: Mycobacterium tuberculosis, BCG, T cells

\section{INTRODUCTION}

Disease caused by Mycobacterium tuberculosis continues to be one of the most prevalent causes of mortality from a bacterial infection in the world and is further exacerbated by the HIV epidemic, the emergence of drug-resistant tuberculosis, and other factors such as malnutrition, immigration and the ease of air travel allowing rapid global transmission (Bloom \& Murray, 1992; Dye et al., 1999; Netto et al., 1999; Horsburgh, 2000; Lillebaek et al., 2001). Despite this, it is paradoxical that almost certainly the majority of individuals tend to be resistant to tuberculosis, and in fact there are numerous studies that suggest that genetic factors influence the capacity of the individual to resist infection with this organism. Various studies point to the possible existence of resistance genes in people from various parts of the world (Shaw et al., 1997; Bellamy et al., 1998, 2000) and certain ethnic groups (Stead et al., 1990). In animal models, the classical studies of Lurie (reviewed by Dannenberg, 1994), who developed resistant and susceptible strains of rabbits, have been more recently followed by various studies in the mouse model which have categorized certain mouse strains in terms of their ability to resist mycobacterial disease.

This led to the identification of a gene locus, Nramp1, which predicts the susceptibility of mouse strains to intravenous infection with low doses of the Montreal substrain of the BCG vaccine (Gros et al., 1981; Vidal et al., 1995). Interestingly, this phenotype is reversed if such animals are infected with $M$. tuberculosis, with, for example, mice on the C57BL/6 background being resistant to tuberculosis infection, whereas other strains such as $\mathrm{DBA} / 2, \mathrm{CBA} / \mathrm{J}$ and $\mathrm{A} / \mathrm{J}$ mice are more susceptible (Medina \& North, 1996, 1998). Such susceptible mouse strains seem to have an intact lung innate response and are able to initially restrain pulmonary infection delivered by aerosol (Medina \& North, 1996, 1998; Turner et al., 2001a), but the subsequent chronic stage of the disease is not stable and these animals succumb approximately 150-200 days later from a form of reactivation disease (Turner et al., 2001a).

We have previously suggested that the root cause of this phenomenon is an inability of $\mathrm{T}$ cells in these susceptible strains to up-regulate the expression of adhesion/inte- 
grin molecules to allow these cells to cross pulmonary blood vessel walls and move through tissues to the sites of infection (Turner et al., 2001a). In the current study, we hypothesized that this defect might be avoided if these mice were either vaccinated with BCG or infected with $M$. tuberculosis and given chemotherapy, thus establishing a pool of memory $\mathrm{T}$ cells that might be better equipped to express such molecules. The results of the study show that such cells can mediate increased resistance in the spleen to a challenge infection, but in susceptible strains are again poorly focused in the lungs after aerosol challenge, and that the cause of this may again be associated with poor expression of these necessary adhesion/integrin molecules. Such defects, if they occur in humans, may contribute to both the inability of the BCG vaccine to protect certain individuals and the triggering of reactivation disease.

\section{METHODS}

Mice. Specific pathogen free 6-8-week-old female C57BL/6, CBA/J and DBA/2 mice were obtained from Jackson Laboratories (Bar Harbour, ME, USA). Infected mice were housed in an ABL-3 biohazard facility and were maintained with sterile bedding, water and mouse chow. Sentinel mice within the colony tested negative for 12 known mouse pathogens.

Bacteria. Mycobacterium bovis BCG Pasteur and M. tuberculosis $\mathrm{H} 37 \mathrm{Rv}$ were originally obtained from the Trudeau Institute, Saranac Lake, NY. Bacteria were grown to midexponential phase in Proskauer-Beck broth and then frozen in aliquots at $-70{ }^{\circ} \mathrm{C}$ until needed. Bacterial c.f.u. $\mathrm{ml}^{-1}$ were determined by plating serial dilutions on Middlebrook $7 \mathrm{H} 11$ agar (Becton Dickinson), supplemented with oleic acidalbumin-glucose complex, and counting the colonies appearing after 21 days incubation at $37^{\circ} \mathrm{C}$. Levels of protection were calculated by subtracting the mean $\log _{10}$ number of bacteria in BCG-vaccinated mice from those in non-vaccinated controls.

Immunization of mice. Mice were vaccinated with $10^{6}$ live $M$. bovis BCG Pasteur bacilli suspended in $200 \mu \mathrm{l}$ sterile saline subcutaneously, in the scruff of the neck. Immunity was allowed to develop for 6 weeks prior to challenge with $M$. tuberculosis H37Rv.

Challenge infections. Mice were infected with $10^{5}$ live $M$. tuberculosis $\mathrm{H} 37 \mathrm{Rv}$ bacilli suspended in $200 \mu \mathrm{l}$ sterile saline intravenously, in the lateral tail vein. For aerosol infections, mice were exposed using a Glas-Col airborne exposure apparatus calibrated to deliver approximately 100 bacteria into the lungs. To generate memory immune mice, isoniazid and rifampicin were added to the drinking water to achieve approximate daily doses of 25 and $10 \mathrm{mg} \mathrm{kg}^{-1}$ per day.

Preparation of splenocytes for flow cytometry. One week following i.v. challenge with $M$. tuberculosis, the mice were killed and the spleens were aseptically removed. Single-cell suspensions of splenocytes were prepared by passing spleen tissue through a $70 \mu \mathrm{m}$ nylon cell strainer and resuspending in Dulbecco's Modification of Eagle's Medium (DMEM) (Fisher Scientific), supplemented with $10 \%$ heat-inactivated fetal calf serum, $2 \mathrm{mM}$ L-glutamine, 0.01 M HEPES, $1 \times$ antibiotic/ antimycotic, $2 \times$ MEM non-essential amino acids and $0.05 \mathrm{mM}$ 2-mercaptoethanol. Chemicals were purchased from Sigma, unless stated otherwise. Cell suspensions were kept on ice during preparation. Cell suspensions were centrifuged, the supernatants decanted and the pellets resuspended in $2 \mathrm{ml}$ lysing buffer $\left(0.15 \mathrm{mM} \mathrm{NH} \mathrm{NH}_{4} \mathrm{Cl}, 1.0 \mathrm{mM} \mathrm{KHCO}, 0.1 \mathrm{mM}\right.$ $\mathrm{Na}_{2}$ EDTA, pH 7.4) for $3 \mathrm{~min}$ at room temperature to deplete the suspension of red blood cells. The cells were then centrifuged, the supernatants decanted and the cell pellets resuspended in RPMI medium deficient in biotin and phenol red (DRPMI) (Irvine Scientific) and containing 0.1\% sodium azide.

Flow cytometry. Cells were incubated with FITC-, phycoerythrin (PE)-, biotin-, allophycocyanin (APC)- or peridinin chlorophyll-a protein (PerCP)-labelled specific antibodies (25 $\mu \mathrm{g} \mathrm{ml}^{-1}$, diluted in DRPMI/azide) for $30 \mathrm{~min}$ at $4{ }^{\circ} \mathrm{C}$ in the dark. Antibodies were purchased from Pharmingen. Biotinlabelled cells were washed once with DRPMI/azide, and then incubated with Red-670-labelled streptavidin (Gibco-BRL) for $30 \mathrm{~min}$ at $4{ }^{\circ} \mathrm{C}$ in the dark. The cells were then washed twice and resuspended in DRPMI/azide. Flow cytometry analysis was performed using a Becton Dickinson FACSCalibur instrument. Cell surface markers included FITC-labelled CD4, CD8, CD44, CD11a and CD54; PE-labelled CD4, CD8, CD44 and CD45RB; biotin-labelled CD45RB, CD62L, CD11a and CD54; PerCP-labelled CD4; and APC-labelled CD8 and CD62L. Lymphocytes were gated on forward and side scatter profiles. CD4 + and CD8 + lymphocytes were identified as cell populations expressing high levels of these respective antigens bound to fluorescent-labelled antibody. Isotype controls were included as part of the experiment. All analyses were performed using CellQuest software (Becton Dickinson).

\section{RESULTS}

\section{Capacity of BCG to protect susceptible and resistant mouse strains after challenge via the intravenous or aerosol routes}

The data shown in Fig. 1 are representative of several experiments in which the influence of the challenge route on subsequent resistance was tested. In such studies it was consistently found that subcutaneous BCG vaccination protected most strains tested almost identically in terms of the reduction in the bacterial load in the spleen after intravenous infection. The data shown here are the conventional day 10 assay, reflecting the peak load in the spleens of control mice. Similar protection was seen in the liver counts (data not shown). In the lungs, however, whilst good protection was seen in the C57BL/6 mouse, very low levels were consistently seen in susceptible mouse strains such as DBA/2 or $\mathrm{CBA} / \mathrm{J}$.

Similarly, minimal protection was seen in susceptible strains (an experiment using the CBA/J strain is shown here as a representative example) if the animals were exposed to low-dose aerosol challenge after BCG vaccination. In both cases, bacterial growth curves demonstrated that these observations were not due to a shift in the kinetics of peak protection in the different mouse strains (data not shown), and did not reflect any major differences in the bacterial load in the lungs of non-vaccinated controls. 

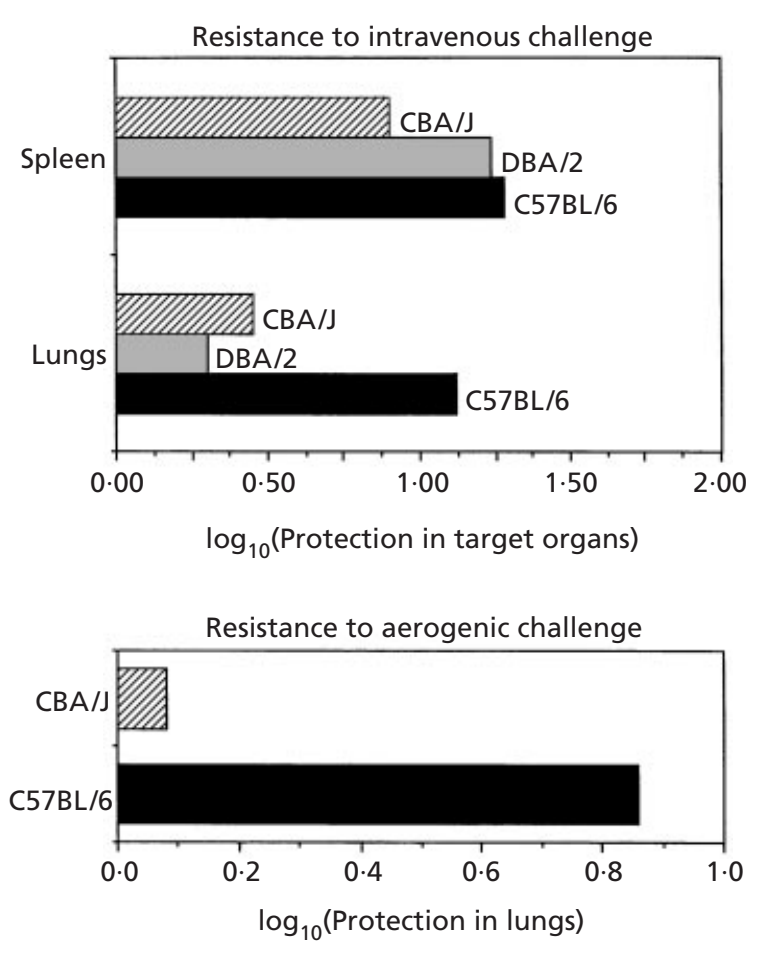

Fig. 1. Comparative resistance of different BCG-vaccinated inbred mouse strains to $M$. tuberculosis delivered via the intravenous or aerosol routes. The data shown are expressed as the $\log _{10}$ differences in the mean bacterial loads of vaccinated versus control mice of each strain $(n=5)$.

\section{Changes in the response by memory T-cell populations in susceptible and resistant strains}

To determine if the apparent inability of susceptible mouse strains to control $M$. tuberculosis in the lungs even after BCG vaccination was due to an inability to generate a memory $\mathrm{T}$-cell response, the number of cells accumulating in the spleens after intravenous infection that expressed a CD4 CD44 ${ }^{\text {hi }} \mathrm{CD} 45 \mathrm{RB}{ }^{\text {lo }}$ phenotype was determined. As shown in Fig. 2, an increase in these cells was observed in both the C57BL/ 6 mice and the DBA/2 mice after challenge, compared to those seen in mice that had not been vaccinated $(P<0 \cdot 05$, Student's $t$ test $)$. A similar trend was also seen in terms of increases in numbers of CD4 cells with a CD62L $\mathrm{L}^{10}$ phenotype, although because here numbers of cells in non-vaccinated mice challenged with $M$. tuberculosis were markedly higher to begin with, these differences were not statistically significant $(P$ values from $0 \cdot 1$ to $0 \cdot 4$, Student's $t$ test).

Given that there is now substantial evidence that CD8 T cells are also important in immunity to tuberculosis (Orme et al., 1992; Flynn et al., 1992; Serbina \& Flynn, 1999; D'Souza et al., 2000; Turner et al., 2001b), changes in CD8 T-cell numbers in the spleen indicative of a potential memory $\mathrm{T}$-cell response were also monitored. The results of these analyses, looking at the changes in the expansion of CD8 CD44 $4^{\text {hi }}$ and CD8 CD62L $\mathrm{L}^{\text {10 }}$ populations, showed that such populations increased in
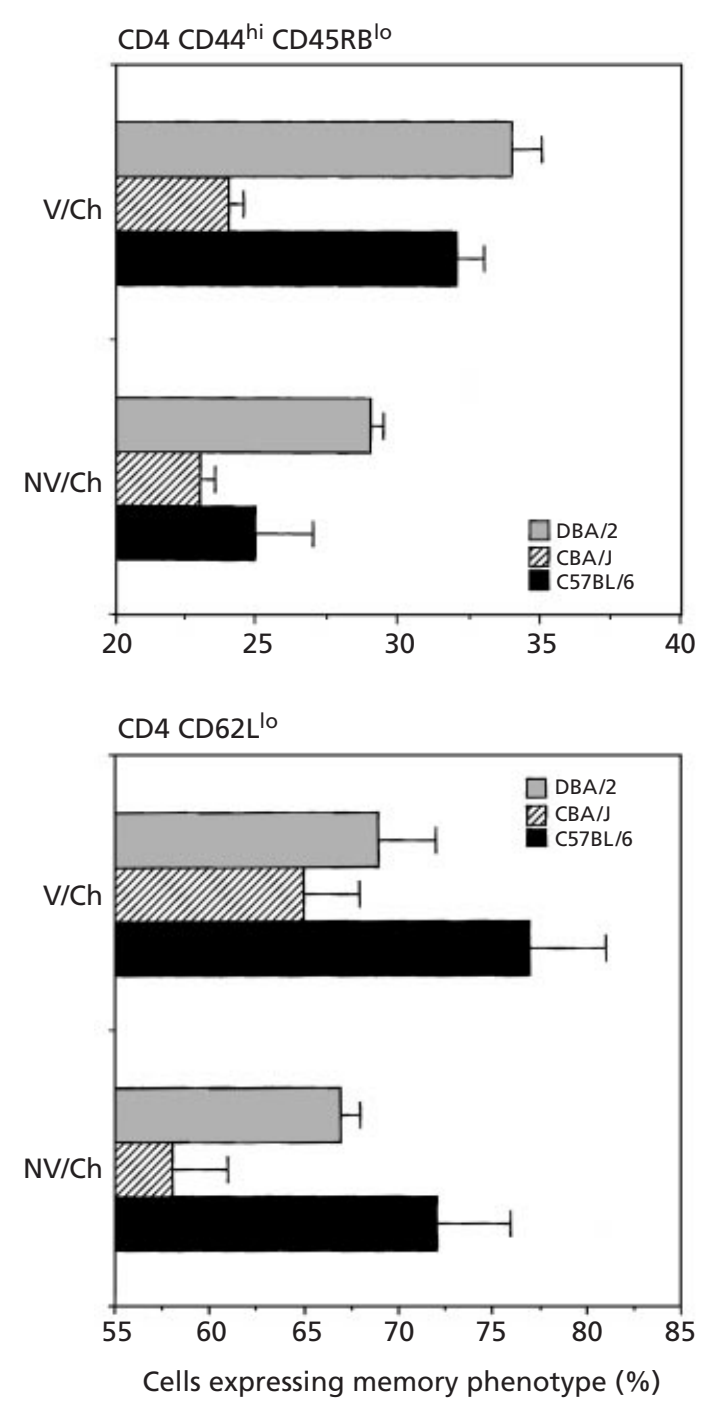

Fig. 2. Percentages of splenic CD4 T cells expressing a memory phenotype in three inbred mouse strains after challenge with $M$. tuberculosis. NV/Ch, naïve mice challenged; V/Ch, BCGvaccinated mice challenged. Data shown are mean flow cytometric values $(n=4) \pm$ SEM.

C57BL/6 mice, but remained much the same in the susceptible CBA/J and DBA/2 strains (Fig. 3).

\section{Expression of the CD11a and CD54 molecules is lower in susceptible mouse strains}

In order for $\mathrm{T}$ cells to enter the lungs, they need to adequately express integrin and adhesion molecules. The data shown in Fig. 4 show that CD4 T cells in all three strains of mouse increased their expression of CD11a if the mice were first vaccinated before challenge, with C57BL/6 mice showing the largest changes $(P=$ $0 \cdot 03$, Student's $t$ test). In terms of CD 54 , a similar change was seen on $\mathrm{T}$ cells from C57BL $/ 6$ mice $(P<0 \cdot 001$, Student's $t$ test), whereas changes on cells from the other mouse strains were not significant. 

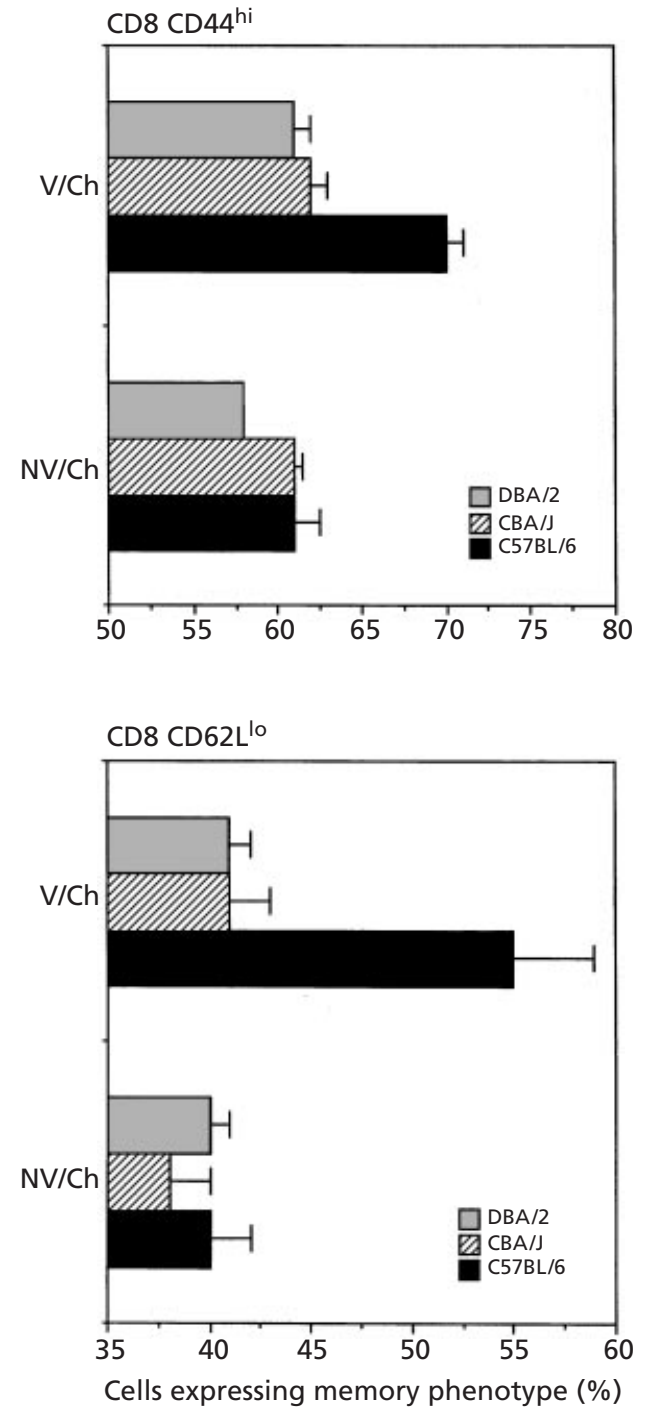

Fig. 3. Percentages of splenic CD8 T cells expressing a memory phenotype in three inbred mouse strains after challenge with $M$. tuberculosis. NV/Ch, naïve mice challenged; V/Ch, BCGvaccinated mice challenged. Data shown are mean flow cytometric values $(n=4) \pm$ SEM.

\section{Differences in cell influx in memory immune mice}

The evidence above indicated that BCG vaccination of susceptible mouse strains only poorly induced increased expression of adhesion and integrin molecules, thus
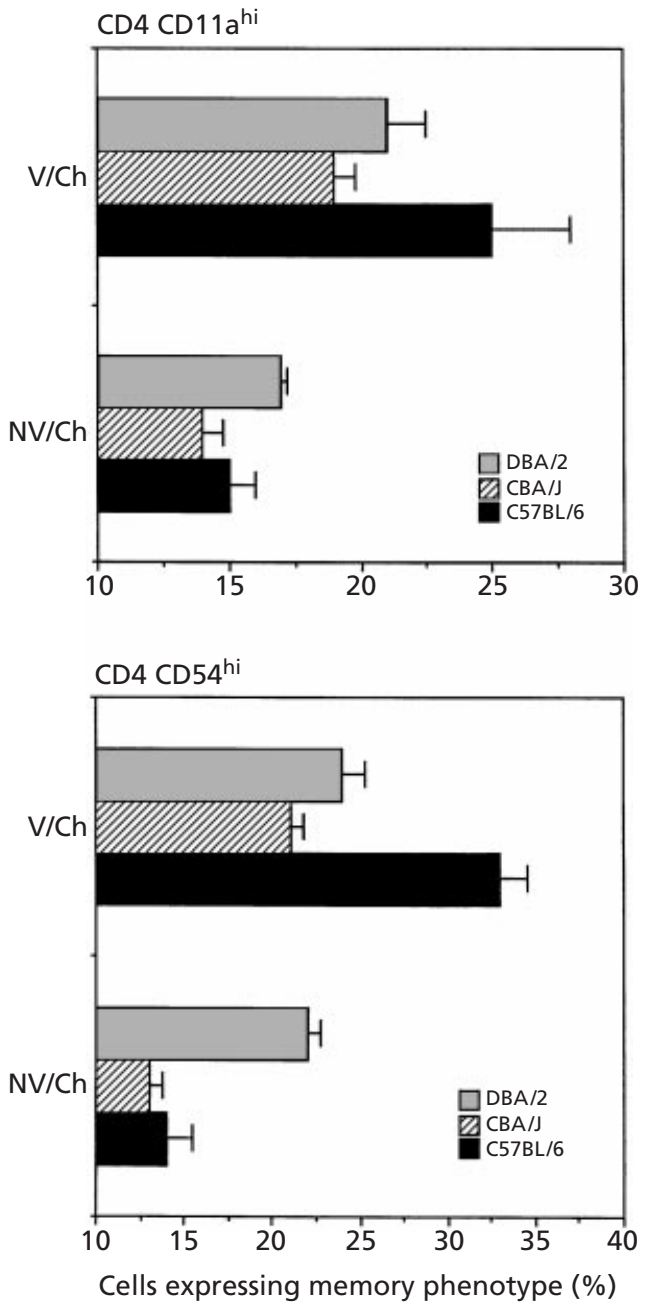

Fig. 4. Percentages of splenic CD4 $T$ cells expressing upregulation of CD11a and CD54 in three inbred mouse strains after challenge with $M$. tuberculosis. NV/Ch, naïve mice challenged; V/Ch, BCG-vaccinated mice challenged. Data shown are mean flow cytometric values $(n=4) \pm$ SEM.

possibly explaining the poor resistance seen in the lungs. In a final series of studies, therefore, we decided to provide a stringent test of this possibility by infecting groups of C57BL/6 and CBA/J mice directly with $M$. tuberculosis followed soon after by chemotherapy, in an attempt to establish a very strong state of memory immunity (Orme, 1988). To date, using this model, we

Fig. 5. Representative histopathological appearance of lungs of naïve or memory immune mice after challenge by aerosol with $M$. tuberculosis. The response in C57BL/6 mice is shown in the left column and that in CBA/J mice is shown in the right column. There was a florid granulomatous response evident in the C57BL/6 mice by day 40 (a), with lesions becoming even larger and more diffuse by day 70 (b). In memory immune mice, the influx of lymphocytes was clearly accelerated with large numbers present on day 40 (c) and with some degree of lesion resolution evident by day 70 (d). In naïve CBA/J mice, in contrast, the granuloma was poorly formed by day 40 (e) and foamy macrophages predominated. Some aggregates of lymphocytes could be seen on day 70 (f) but these were localized within the field of macrophages. In memory immune mice, a substantial influx of lymphocytes was seen on day 40 (g) but these were mostly in the form of perivascular cuffs with very few entering the adjacent lesions. By day 70, the lesions were very large and lymphocyte aggregations could now be seen (h). However, there were also large areas of cellular degeneration containing clusters of neutrophils (arrowed). Bar, $100 \mu \mathrm{m}$. Haematoxylin and eosin staining. 


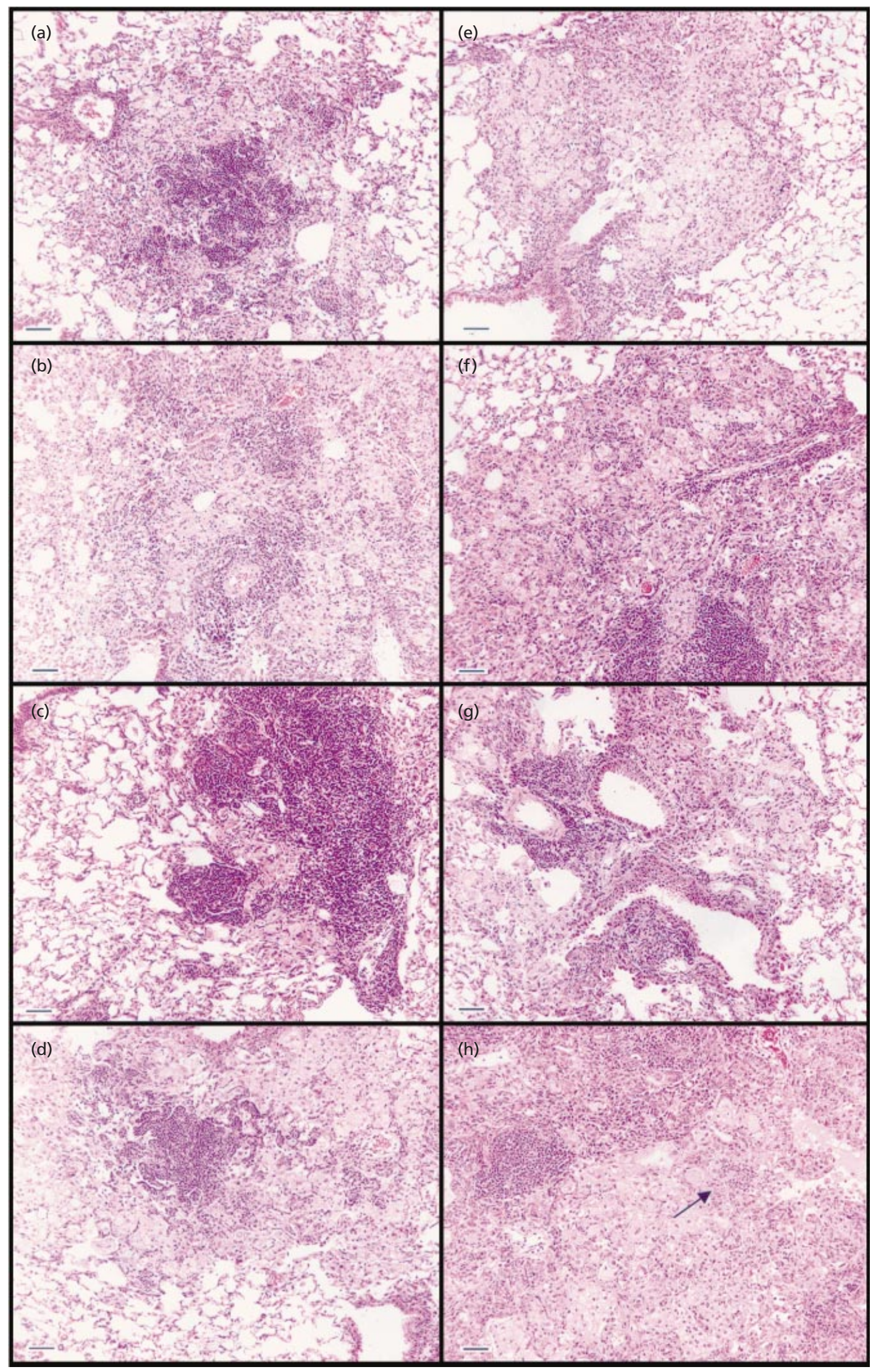

Fig. 5. For legend see facing page. 
have not seen intrinsic differences in memory $\mathrm{T}$ cells induced by BCG or by M. tuberculosis infections.

Representative data are shown in Fig. 5. Forty days after aerosol exposure of naïve mice, a substantial granulomatous response was evident in the lungs of C57BL/6 mice (Fig. 5a) and by day 70 these lesions were generally larger, if somewhat more diffuse in terms of lymphocyte densities (Fig. 5b). In memory immune mice, evidence of an accelerated response was suggested by a florid rapid influx of lymphocytes clearly evident by day 40 (Fig. 5c). By day 70 , such lesions remained organized in nature, and appeared to be resolving (Fig. 5d).

In CBA/J mice, lesions on day 40 were more diffuse and the cellular influx was dominated by macrophages with minimal lymphocyte infiltration (Fig. 5e). By day 70 , this appearance had now changed and aggregates of lymphocytes were now discernible (Fig. 5f). In CBA/J mice previously infected with $M$. tuberculosis, cell influx was improved compared to naïve mice, but, interestingly, most lymphocytes entering the lungs tended to accumulate around blood vessels or along side airways (Fig. 5g). Probably directly as a result of this mostly perivascular distribution of incoming lymphocytes, by day 70 lung lesions were very large and dominated by fields of vacuolated macrophages, with small pockets of neutrophils (Fig. 5h, arrow) indicating local tissue destruction and necrosis.

\section{DISCUSSION}

The results of this study show that susceptible strains of mice are as resistant as the C57BL/6 strain if vaccinated with BCG and then challenged intravenously with $M$. tuberculosis, in terms of the subsequent reduction in the bacterial load in the spleen and liver. In contrast, despite this, susceptible strains were poorly protected in terms of their capacity to reduce the bacterial load in the lungs, regardless of whether the challenge infection was delivered by the intravenous or aerogenic routes.

It is thoroughly established in the literature that CD4 $\mathrm{CD} 44^{\text {hi }} \mathrm{CD} 4 \mathrm{RB}^{\text {lo }} \mathrm{T}$ cells mediate immunological memory, and it was interesting to note that $T$ cells in the C57BL/ 6 mice showed the highest percentage change in their expression. A higher initial number was seen in DBA/2 mice that had not been vaccinated, and this also increased. In contrast, there was no significant change in the levels of cells of this phenotype in the CBA/J mice. Interesting, this unusually high level of (apparently) memory $\mathrm{T}$ cells in the DBA/2 strain persists with age and is prominent in old mice (J. Turner \& I. M. Orme, unpublished).

The results also show that vaccination with BCG followed by challenge induced the expansion of memory CD8 $\mathrm{T}$ cells in the C57BL/6 mice, whereas minimal changes were seen in the two susceptible strains tested. This may imply that the rapid resistance seen in vaccinated C57BL/6 mice may in fact involve a substantial CD8 component, and experiments to determine this are currently planned.
Finally, the results of this study show that there were obvious differences in terms of histological data regarding the lymphocytic influx into the lungs of resistant and susceptible mice, even if such mice were previously infected with M. tuberculosis to induce a strong state of memory immunity. In the C57BL/6 mouse, there was an efficient influx of lymphocytes and this was clearly amplified and accelerated in the memory immune mice. In contrast, in the susceptible CBA/J strain, there was evidence for a slower lymphocyte influx, leading to lesions that were dominated by macrophages instead. In memory immune mice, there appeared to be a much faster arrival of lymphocytes into the lungs, but it was clearly evident that most of these cells were accumulating around blood vessels or airways and were failing to move into the lung tissues.

Our working hypothesis to date has been that susceptible mouse strains such as the $\mathrm{CBA} / \mathrm{J}$ and DBA/2 strains have the same underlying mechanisms that make them poorly resistant to lung infection with $M$. $t u$ berculosis and unable to eventually prevent reactivation disease (Turner et al., 2001a). However, at least in terms of CD4 memory populations, there appear to be clear differences between these strains, with DBA/2 mice seeming to have higher numbers of memory phenotype CD4 cells even without BCG vaccination, while CBA/J mice, in contrast, seem to have a minimal capacity to mount a memory $\mathrm{T}$-cell response even when first given BCG.

In naïve susceptible mice, a reduced ability to focus $\mathrm{T}$ cells into the lungs has been demonstrated (Turner $\mathrm{et}$ al., 2001a) to possibly underlie their propensity to develop reactivation tuberculosis. This supports the concept that $\mathrm{T}$ cells pass into the sinusoidal spleen tissues readily in both susceptible and resistant mouse strains, explaining the equal protection seen in the spleen to intravenous challenge, but the lack of strong expression of adhesion molecules in the susceptible strains precludes them from crossing the arteriolar endothelial wall sufficiently well enough to rapidly accumulate in lesions where immunity needs to be expressed. Thus it appears that in the susceptible strains T cells circulate well through lymphoid tissues, but poorly into inflammatory sites such as the lungs.

The hypothesis tested in the current study was the possibility that this defect could be avoided by procedures designed to establish a strong state of memory immunity. Whereas the levels of CD54 expression increased in all three strains (although to only a small degree in $\mathrm{DBA} / 2$ mice), it was found that both $\mathrm{CBA} / \mathrm{J}$ and DBA/2 mice were capable of up-regulating expression of the integrin CD11a. However, whereas the expression of the integrin CD11a increased from $14.5 \%$ to $25 \%$ on CD4 T cells in C57BL/6 mice, in CBA/J the response was lower, increasing from $14 \%$ to $18 \%$. Although strictly speaking this is unproven, this appears to be consistent with the subsequent histology data and may thus explain why lymphocytes in the CBA/J mouse could cross the blood vessel wall (CD54-dependent) but not advance any further (integrin-dependent). 
Returning to the issue of whether the susceptible strains of mice can generate memory $\mathrm{T}$ cells, the overall picture seems to suggest that the basis of the susceptibility of these mice is complex and may involve a number of parameters controlling the generation and further expansion of memory $T$ cells, in addition to their capacity to focus efficiently in the lungs after pulmonary infection. The fact that these changes (or lack of them) extend to the CD8 population as well as CD4 cells is particularly interesting, given the growing realization that CD8 cells may play an important role in resistance to tuberculosis (Orme et al., 1992; Flynn et al., 1992; Serbina \& Flynn, 1999; D'Souza et al., 2000; Turner et al., 2001b), especially during the chronic phase of the disease. In this regard, C57BL/6 mice are normally resistant to reactivation of the chronic disease state (Turner et al., 2001a), but disease is reactivated in the mice if their CD8 gene is disrupted (Turner et al., 2001b).

Variations between individuals in terms of their establishment of an effective memory T-cell pool after either BCG vaccination or successful control earlier in life of a primary exposure to tuberculosis may underlie their risk of subsequently developing reactivation tuberculosis. This risk would then be further influenced by various extrinsic factors, such as diabetes, malnutrition, immunosuppression and so forth, that may tip the balance between control of the infection and potential regrowth. Regardless, however, if the results from the mouse studies can be extrapolated to human beings, they may imply that BCG vaccination may be intrinsically more effective in one individual as compared to another, with a functionally weaker memory response in the latter rendering the individual more susceptible to primary infection or reactivation of previously latent disease, with this susceptibility further compounded by the capacity of the cell to express molecules that enable it to cross blood vessels and/or to move through the extracellular matrix. As suggested by both the various mouse studies and clinical studies, predisposition to these events almost certainly has a genetic basis.

In conclusion, it is interesting to note that there is compelling evidence in certain clinical trials that suggests that BCG is still having some immunological effect even though it failed to protect against tuberculosis. One such example is the trial in Northern Malawi (Fine et al., 1986) in which BCG had no effect whatsoever against the incidence of tuberculosis but had a significant protective effect against the incidence of leprosy. Since the anatomical sites of the two diseases are different, it is tempting to speculate that in the 'susceptible' Malawi population, immune $T$ cells focus poorly in the lungs but are still capable of entering lesions in the periphery caused by M. leprae.

\section{ACKNOWLEDGEMENTS}

This work was supported by US Public Health Service grants AI-45707 and AI-44072 from the NIH. We thank Dr Paul Fine for an interesting discussion.

\section{REFERENCES}

Bellamy, R., Ruwende, C., Corrah, T., McAdam, K. P., Whittle, H. C. \& Hill, A. V. (1998). Variations in the NRAMP1 gene and susceptibility to tuberculosis in West Africans. N Engl J Med 338, 640-644.

Bellamy, R., Beyers, N., McAdam, K. P. \& 14 other authors (2000). Genetic susceptibility to tuberculosis in Africans: a genome-wide scan. Proc Natl Acad Sci U S A 97, 8005-8009.

Bloom, B. R. \& Murray, C. J. (1992). Tuberculosis: commentary on a reemergent killer. Science 257, 1055-1064.

D'Souza, C. D., Cooper, A. M., Frank, A. A., Ehlers, S., Turner, J., Bendelac, A. \& Orme, I. M. (2000). A novel nonclassic beta2microglobulin-restricted mechanism influencing early lymphocyte accumulation and subsequent resistance to tuberculosis in the lung. Am J Respir Cell Mol Biol 23, 188-193.

Dannenberg, A. M., Jr (1994). Roles of cytotoxic delayed-type hypersensitivity and macrophage-activating cell-mediated immunity in the pathogenesis of tuberculosis. Immunobiology 191, 461-473.

Dye, C., Scheele, S., Dolin, P., Pathania, V. \& Raviglione, M. C. (1999). Consensus statement. Global burden of tuberculosis: estimated incidence, prevalence, and mortality by country. WHO Global Surveillance and Monitoring Project. JAMA ( $\mathrm{Am}$ Med Assoc) 282, 677-686.

Fine, P. E., Ponnighaus, J. M., Maine, N., Clarkson, J. A. \& Bliss, L. (1986). Protective efficacy of BCG against leprosy in Northern Malawi. Lancet 2, 499-502.

Flynn, J. L., Goldstein, M. M., Triebold, K. J., Koller, B. \& Bloom, B. R. (1992). Major histocompatibility complex class I-restricted $\mathrm{T}$ cells are required for resistance to Mycobacterium tuberculosis infection. Proc Natl Acad Sci U S A 89, 12013-12017.

Gros, P., Skamene, E. \& Forget, A. (1981). Genetic control of natural resistance to Mycobacterium bovis (BCG) in mice. J Immunol 127, 2417-2421.

Horsburgh, C. R., Jr (2000). The global problem of multidrugresistant tuberculosis : the genie is out of the bottle. JAMA ( $\mathrm{J} \mathrm{Am}$ Med Assoc) 283, 2575-2576.

Lillebaek, T., Andersen, A. B., Bauer, J., Dirksen, A., Glismann, S., de Haas, P. \& Kok-Jensen, A. (2001). Risk of Mycobacterium tuberculosis transmission in a low-incidence country due to immigration from high-incidence areas. J Clin Microbiol 39, 855-861.

Medina, E. \& North, R. J. (1996). Evidence inconsistent with a role for the $B c g$ gene (Nramp1) in resistance of mice to infection with virulent Mycobacterium tuberculosis. J Exp Med 183, 1045-1051.

Medina, E. \& North, R. J. (1998). Resistance ranking of some common inbred mouse strains to Mycobacterium tuberculosis and relationship to major histocompatibility complex haplotype and Nramp1 genotype. Immunology 93, 270-274.

Netto, E. M., Dye, C. \& Raviglione, M. C. (1999). Progress in global tuberculosis control 1995-1996, with emphasis on 22 highincidence countries. Global Monitoring and Surveillance Project. Int J Tuberc Lung Dis 3, 310-320.

Orme, I. M. (1988). Characteristics and specificity of acquired immunologic memory to Mycobacterium tuberculosis infection. J Immunol 140, 3589-3593.

Orme, I. M., Miller, E. S., Roberts, A. D., Furney, S. K., Griffin, J. P., Dobos, K. M., Chi, D., Rivoire, B. \& Brennan, P. J. (1992). T lymphocytes mediating protection and cellular cytolysis during the course of Mycobacterium tuberculosis infection. Evidence for different kinetics and recognition of a wide spectrum of protein antigens. J Immunol 148, 189-196. 
Serbina, N. V. \& Flynn, J. L. (1999). Early emergence of CD8(+ ) T cells primed for production of type 1 cytokines in the lungs of Mycobacterium tuberculosis-infected mice. Infect Immun 67, 3980-3988.

Shaw, M. A., Collins, A., Peacock, C. S. \& 7 other authors (1997). Evidence that genetic susceptibility to Mycobacterium tuberculosis in a Brazilian population is under oligogenic control: linkage study of the candidate genes NRAMP1 and TNFA. Tuber Lung Dis 78, 35-45.

Stead, W. W., Senner, J. W., Reddick, W. T. \& Lofgren, J. P. (1990). Racial differences in susceptibility to infection by Mycobacterium tuberculosis. N Engl J Med 322, 422-427.

Turner, J., Gonzalez-Juarrero, M., Saunders, B. M., Brooks, J. V., Marietta, P., Ellis, D. L., Frank, A. A., Cooper, A. M. \& Orme, I. M. (2001a). Immunological basis for reactivation of tuberculosis in mice. Infect Immun 69, 3264-3270.

Turner, J., D'Souza, C. D., Pearl, J. E., Marietta, P., Noel, M., Frank, A. A., Appelberg, R., Orme, I. M. \& Cooper, A. M. (2001b). CD8and CD95/95L-dependent mechanisms of resistance in mice with chronic pulmonary tuberculosis. Am J Respir Cell Mol Biol 24, 203-209.

Vidal, S., Tremblay, M. L., Govoni, G. \& 7 other authors (1995). The Ity/Lsh/Bcg locus: natural resistance to infection with intracellular parasites is abrogated by disruption of the Nramp1 gene. J Exp Med 182, 655-666.

Received 24 January 2002; revised 4 March 2002; accepted 22 March 2002. 